\title{
Gleichheit und Gerechtigkeit
}

\section{Giovanni Fantacci}

Dr. med., Allgemeine Innere Medizin, Hausarzt und Arzt bei der Stiftung Vivendra (Stiftung für Kinder, Jugendliche und Erwachsene mit Beeinträchtigungen)

Bilder sagen mehr als tausend Worte. Das untenstehende Bild provoziert eine ethische Debatte. Die Begriffe Gleichheit und Gerechtigkeit sind zentrale Begriffe in der ethischen Diskussion. Was heisst aber Gleichheit? Was ist Gerechtigkeit? Man würde meinen, es sei doch alles klar. Fangen wir mit der Gleichheit an und schauen uns an, was in den Verfassungen dazu steht. Die Verfassungen der heutigen westlichen Demokratien sind Entwicklungen der Aufklärung, deren bekannteste Forderung diejenige nach Gleichheit, Freiheit und Brüderlichkeit war.

\section{Was ist Gleichheit?}

Gemäss Artikel 8 der Schweizerischen Bundesverfassung sind alle Menschen vor dem Gesetz gleich (Absatz 1). Diese grundsätzliche Rechtsgleichheit wird im folgenden Absatz noch präzisiert:

«Niemand darf diskriminiert werden, namentlich nicht wegen der Herkunft, der Rasse, des Geschlechts, des Alters, der Sprache, der sozialen Stellung, der Le-

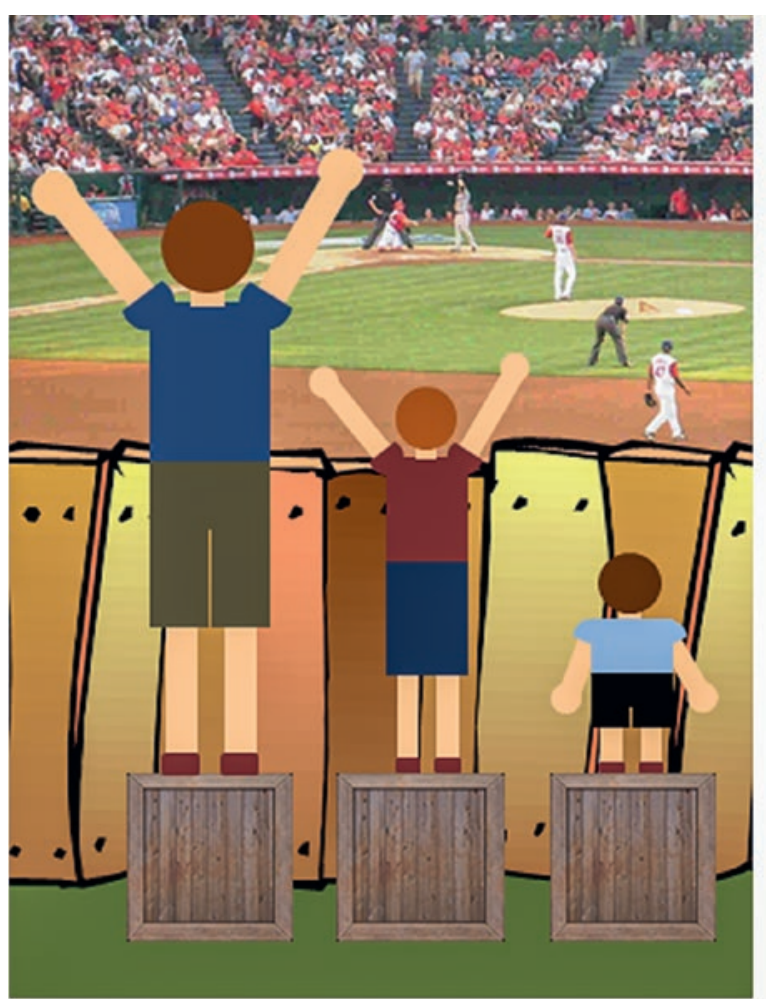

Gleichheit ist nicht gleichbedeutend mit Gerechtigkeit. bensform, der religiösen, weltanschaulichen oder politischen Überzeugung oder wegen einer körperlichen, geistigen oder psychischen Behinderung.»

Noch grundlegender für das Verständnis von Gleichheit und Gerechtigkeit ist der Begriff der Menschenwürde, der in der Bundesverfassung in Artikel 7 definiert ist: Die Würde des Menschen ist zu achten und zu schützen. Im Grundgesetz für die Bundesrepublik Deutschland (23. Mai 1949) wird die Würde im ersten

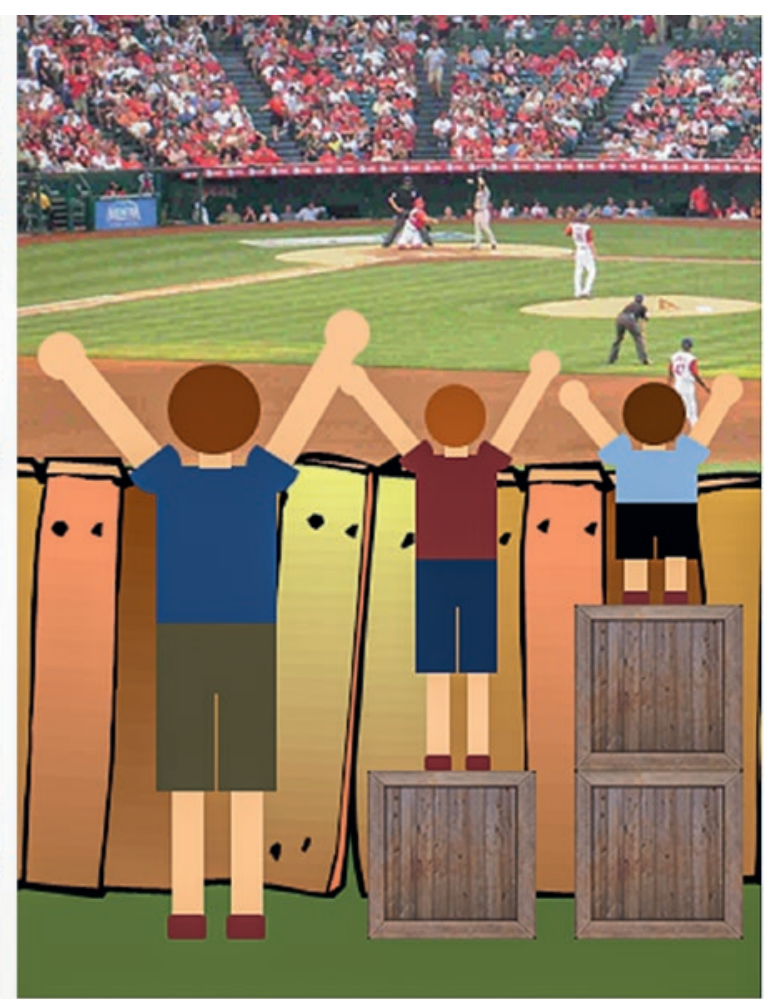


Artikel definiert: Die Würde des Menschen ist unantastbar. Sie zu achten und zu schützen ist Verpflichtung aller staatlichen Gewalt.

Die Würde ist allen Menschen durch die Geburt gegeben. Diese kann auch nicht abgesprochen werden durch Krankheit, Behinderung oder ein anderes Ereignis. Der Rechtsstaat gründet auf einer Rangfolge von Prinzipien, deren Basis die menschliche Würde ist, sie ist die tiefste Grundlage von Ansprüchen auf Freiheit, Gleichheit, Gerechtigkeit und Solidarität. Und da sind schon wieder unsere diskutierten ethischen Werte. Gleichheit könnte heissen, dass alle Menschen bei gleicher Krankheit gleich behandelt werden. Alle haben den Anspruch auf die gleichen Ressourcen. Die Ressourcen sind aber beschränkt, dies wird beispielsweise in der Transplantationsmedizin offensichtlich, wo eine zu geringe Zahl von Transplantationsorganen auf eine grosse Zahl von Kranken trifft, die dringend darauf angewiesen wären. Gleichheit geht ohne die Betrachtung von Gerechtigkeit nicht. So eröffnet sich das weite Feld, wem zuerst geholfen werden soll. Hier hat sich die klinische Ethik entwickelt mit den wichtigen Prinzipien der Entscheidungsfindung (Beauchamp und Childress 2001 in Principles of Biomedical Ethics): Selbstbestimmung oder Autonomie, Nicht-Schaden, Nutzen und Gerechtigkeit. Ich möchte hier auf den Begriff der Gerechtigkeit eingehen.

\section{Was heisst Gerechtigkeit?}

Die ausgleichende Gerechtigkeit oder Tauschgerechtigkeit ist dasjenige, was man dem anderen im arithmetischen Sinn schuldet. Das betrifft die Beziehungen der Menschen zueinander. Die zuteilende Gerechtigkeit ist das, was vom sozialen Ganzen dem Einzelnen zukommt. Es geht also um die Beziehung des Ganzen (Gemeinwohl) zu den Einzelpersonen. Die legale Gerechtigkeit orientiert sich daran, was die Glieder einer Gesellschaft sich im Sinne des Gesetzes gegenseitig schulden.

Grob lassen sich vier Modelle der Gerechtigkeit ausmachen (Giovanni Maio: Mittelpunkt Mensch. Ethik in der Medizin. 2012).

Das Gleichheitsmodell (Egalitarismus): Nach dieser Theorie wäre der zentrale Massstab einer gerechten Zuteilung die Gleichverteilung. Patienten mit gleichem Krankheitsbild sollten den gleichen Zugang zur gleichen Behandlung erhalten, damit keine Ungerechtigkeit entsteht. Im Falle eines Herzinfarktes sollen alle Patienten in den Genuss der modernen Behandlungsmethoden kommen. Es ist offensichtlich, dass dieses Modell nur funktioniert, solange genug Ressourcen vorhanden sind. In der Transplantationsmedizin funk- tioniert dieses Modell nicht mehr. Dort muss eine Auswahl getroffen werden.

Das zweite Modell ist das Freiheitsmodell (Liberalismus). Gerechtigkeit wird hier nach dem Maximum an Freiheit gemessen. Jeder Mensch hat die Freiheit, selber zu entscheiden, welche Leistungen er versichern möchte. Nach diesem Prinzip funktionieren Privatversicherungen. Der Markt und die ökonomischen Verhältnisse bestimmen hier die Verteilung der medizinischen Güter, und es besteht die Gefahr einer Zweiklassenmedizin. Solange das Freiheitsmodell auf medizinische Güter aus nicht zentralen Bereichen, z.B. Akupunktur oder andere alternative medizinische Angebote, beschränkt wird, bestehen keine Probleme.

Beim dritten Modell (Effizienzmodell) ist das Kriterium für eine gerechte Zuteilung die Effizienz. Unter Effizienz versteht man ein gutes Verhältnis von Nutzen und Kosten. Bei neuen Behandlungsmöglichkeiten, die sehr teuer sind, wird diese Frage schon heute diskutiert. Es kann dazu führen, dass nicht alle Patienten behandelt werden, da das Kosten-Nutzen-Verhältnis nicht immer stimmt. Ein Beispiel hierfür sind teure Krebsbehandlungen, die nur eine kurze Lebensverlängerung im Bereich von wenigen Monaten bringen Moderne Gentherapien sind sehr teuer. So kostet Zolgensma zur Behandlung der spinalen Muskelatrophie von Kindern 2,1 Millionen Dollar pro Einmaldosis. Wenn eine Krankheit aber dadurch geheilt werden kann, ist der Preis durchaus gerechtfertigt.

Beim vierten Modell (Fairnessmodell) des amerikanischen Philosophen John Rawls (A Theory of Justice, 1971) hat die Zuteilungsart Vorrang, die auf eine Verbesserung der Gesundheit derjenigen zielt, denen es am schlechtesten geht. Das entscheidende Kriterium ist hier also die Bedürftigkeit. Ein Beispiel wäre die Transplantationsmedizin: Dort wird anhand von bestimmten Kriterien eine Art Rangliste erstellt, wer zuerst Anspruch auf ein Spenderorgan haben soll. In einer ethischen Debatte müssen die Begriffe mit Inhalt gefüllt werden. Gerade in der modernen Medizin mit neuen, zum Teil sehr teuren Therapien braucht es einen vernünftigen Dialog darüber, was sinnvoll ist. Gerechtigkeit muss immer genau definiert werden. Der ethische Dialog besteht aus einem Abwägen der verschiedenen Argumente. Es gibt keine einfachen Antworten auf die heutigen komplexen Herausforderungen. Wir kommen dann zu einer guten Lösung ethischer Konflikte, wenn man versucht, die vier Gerechtigkeitsmodelle in einem ausgewogenen Verhältnis nebeneinander als allesamt berechtigte und zugleich notwendige Modelle zu betrachten.

Bildnachweis

(C) Craig Froehle | University Cincinnati 